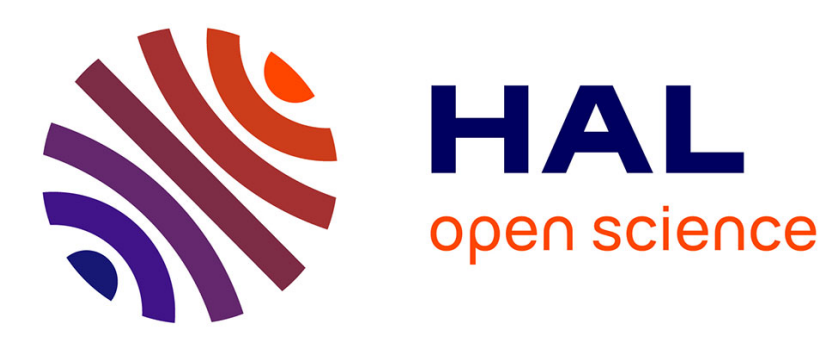

\title{
Is visual fatigue changing the perceived depth accuracy on an autostereoscopic display?
}

Marcus Barkowsky, Romain Cousseau, Patrick Le Callet

\section{To cite this version:}

Marcus Barkowsky, Romain Cousseau, Patrick Le Callet. Is visual fatigue changing the perceived depth accuracy on an autostereoscopic display?. SPIE 2011 Stereoscopic Displays and Applications XXII, Jan 2011, San Francisco, United States. pp.7863, 78631V, 10.1117/12.872572 . hal00616686v2

\section{HAL Id: hal-00616686 \\ https://hal.science/hal-00616686v2}

Submitted on 24 Aug 2011

HAL is a multi-disciplinary open access archive for the deposit and dissemination of scientific research documents, whether they are published or not. The documents may come from teaching and research institutions in France or abroad, or from public or private research centers.
L'archive ouverte pluridisciplinaire HAL, est destinée au dépôt et à la diffusion de documents scientifiques de niveau recherche, publiés ou non, émanant des établissements d'enseignement et de recherche français ou étrangers, des laboratoires publics ou privés. 


\title{
Is visual fatigue changing the perceived depth accuracy on an autostereoscopic display?
}

\author{
Marcus Barkowsky, Romain Cousseau, Patrick Le Callet \\ IRCCyN UMR no 6597 CNRS, University of Nantes, Nantes, France
}

\begin{abstract}
In this paper, a subjective study is presented which aims to measure the minimum perceivable depth difference on an autostereoscopic display in order to provide an indication for visual fatigue. The developed experimental setup was used to compare the subject's performance before and after 3D excitation on an autostereoscopic display. By comparing the results to a verification session with $2 \mathrm{D}$ excitation, the effect of $3 \mathrm{D}$ visual fatigue can be isolated. It was seen that it is possible to reach the threshold of acuity for stereo disparity on that autostereoscopic display. It was also found that the measured depth acuity is slightly higher after $3 \mathrm{D}$ viewing than after $2 \mathrm{D}$ viewing.
\end{abstract}

Keywords: 3DTV, Visual Fatigue, Depth perception, Autostereoscopic display, Subjective experiment

\section{INTRODUCTION}

Recently, 3DTV services have been introduced to the public together with a variety of stereoscopic and autostereoscopic displays. Shutter glasses based stereoscopic systems are currently most frequently used at the home but in the near future, autostereoscopic displays might become available that offers a sufficiently high resolution and that benefits from the fact that wearing eye-glasses is not mandatory to see the $3 \mathrm{D}$ effects.

At the moment, it is often considered that one of the major risks in the wide acceptance of 3D television is visual discomfort and visual fatigue. Some sources of immediate visual discomfort have been identified, e.g. the vergence/accommodation conflict [1], rapid changes in horizontal disparity [2][3], and objects moving regularly in depth, e.g. continuous changes of horizontal disparity [4]. Recent surveys on the most likely causes of visual fatigue and visual discomfort can be found in [5][6].

Several methods have been tested in order to measure visual fatigue with questionnaires [7] and to identify observers that are particularly sensitive to it [8]. Other alternative have been tested, ranging from a task performance measurement [9] to analyzing an Electroencephalogram (EEG) [10]. However, so far, no reliable method has been found that is simple to apply to a large panel of naîve observers.

The human visual system (HVS) is capable of automatically controlling the accommodation and vergence of the eyes. In fact, each change in accommodation immediately triggers a change in vergence and vice versa. The rate at which one system influences the other is known as the accommodation/convergence ratio $(\mathrm{CA} / \mathrm{C})$ and convergence/accommodation ratio (AC/A). A recent detailed study on the relationship between the two ratios and the individual difference that might occur can be found in [11]. The experimental setup is similar to the situation found on stereoscopic flat panel displays. It was seen that a significant overshoot of the accommodation/vergence system is seen for some subjects and that the stereo presentation might lead to a conflict between the two coupled adaptation mechanisms AC/A and CA/C.

This seems in line with an experiment that we conducted earlier [9]. We conducted an optometric fusional vergence facility test at near [12] before and after viewing the same content in 2D and 3D presentation mode. In this optometric prism facility test, the ability of a person is measured to change its convergence voluntarily because he needs to compensate for the offset induced by a prism in his visual pathway. This decoupling ability may be seen as inhibiting to a certain extent the automatic adaptation ability measured in the AC/A and CA/C factors. The speed of correct convergence was faster after $3 \mathrm{D}$ viewing than after $2 \mathrm{D}$ viewing. One possible reason is that this might be due to the unnatural viewing condition: The observer learns how to decouple the convergence system from the accommodation. 
It might be anticipated that the ability of changing the vergence independently from the accommodation reduces the acuity of the HVS to depth differences. This will be evaluated in this paper by displaying stimuli that only exhibit disparity information and by asking the observer to indicate whether a difference between two stimuli is visible and thus above their depth acuity threshold.

The paper is organized as follows: In Section 2, the subjective experiment method will be explained and in Section 3 the results of the study will be presented including a detailed discussion of the results before concluding the paper in the last section.

\section{SUBJECTIVE EXPERIMENT SETUP}

The subjective experiment was split into two sessions. Each observer participated in both sessions which were separated by at least three days in order to avoid memorization effects.

The overall setup of the sessions is depicted in Figure 1. A total of ten naïve observers participated in the experiment. All observers started with the 3D session (session A). The 2D session (session B) only differs in the excitation part. In order to excite the observers with 3D content and to assure that they stayed focused on the display, the observers were playing a simple car racing videogame (flatout). As the own car was displayed coming out in front of the screen, this setup exhibited visual fatigue in the 3D viewing case. In the second session which was separated by at least three days from the first session, the same setup was used except that they played in 2D instead of 3D.

At the beginning of their respective first session, they were screened for visual acuity using a Snellen Chart, for depth perception using a Randot Stereo Test, and for correct color vision using Ishihara plates. Their dominating eye was also determined in two ways, firstly using the room corner as a fix-point for covering with the thumb (far vision) and taking the view-finder of a camera close to the dominating eye (near vision). For all our observers, the two tests agreed about the position of the dominating eye. In total, there were 5 right-eye dominant and 5 left-eye dominant viewers.

\begin{tabular}{|c|c|c|c|c|c|c|}
\hline \multirow{2}{*}{ Session A (3D): } & $\begin{array}{l}\text { Prism } \\
\text { Facility }\end{array}$ & Depth Exp. & 3D Excitation & Pause & Depth Exp. & $\begin{array}{l}\text { Prism } \\
\text { Facility }\end{array}$ \\
\hline & $5 \mathrm{~min}$ & $15 \mathrm{~min}$ & $20 \min$ & $5 \mathrm{~min}$ & $15 \min$ & $5 \mathrm{~min}$ \\
\hline \multirow{2}{*}{ Session B (2D): } & $\begin{array}{l}\text { Prism } \\
\text { Facility }\end{array}$ & Depth Exp. & 2D Excitation & Pause & Depth Exp. & $\begin{array}{l}\text { Prism } \\
\text { Facility }\end{array}$ \\
\hline & $5 \mathrm{~min}$ & $15 \mathrm{~min}$ & $20 \mathrm{~min}$ & $5 \mathrm{~min}$ & $15 \mathrm{~min}$ & $5 \mathrm{~min}$ \\
\hline
\end{tabular}

Figure 1: Session setup

The display that was used for displaying the stimulus was the Philips 42" autostereoscopic display. This display was used for the depth experiments and for the 2D and 3D excitation. By using a lenticular array foil in front of an HDTV panel, this display is able to produce nine distinct views in horizontal direction. In order to facilitate the rendering of the nine views, it contains a rendering unit that takes a texture and a depth image as input. The advantage is that the user does not need to generate the nine views himself but the disadvantage is that the influence on the final rendering of the screen can not be fully controlled. In particular, we have already shown that, in a direct comparison, observers prefer to view $2 \mathrm{D}$ presentation to 3D presentation on this screen for most contents [14]. As it was seen that the display processing adds blur to planes that are far away from the screen plane (either in the foreground or in the background), this might influence the results of the depth test as it plays the role of an additional monocular depth cue. Thus, it was decided to use only a small disparity offset in the depth experiment.

\subsection{Optometric test: Fusional vergence facility test at near}

The excitation session was preceded and followed by an examination of the fusional vergence facility using a prism test: Each observer viewed a vertical line of letters at a comfortable reading distance of about $40 \mathrm{~cm}$. A prism of 12 diopters outwards was then introduced into the view of one eye which led to double vision. The participant was asked to fuse the 
two lines again into one. As soon as he succeeded, he changed the prism to 3 diopters inwards and again had to fuse the two appearing lines of characters. After a training phase, the number of full cycles was counted for one minute.

\subsection{Stimulus for the depth experiment}

In the experiment, the observer was asked to compare the position in depth of two plates textured with a Maltese Cross. The stimulus itself was chosen to contain only disparity information and no additional depth cues. In order to support the depth impression of the observers, the rest of the scene contained some depth information. The stimulus is shown in Figure 2. On the left side, the texture is displayed and on the right side, the 8-bit depth map coded into luminance values can be seen. In total, for symmetry reasons, four plates with a Maltese Cross were displayed but only the upper two were used. As can be seen from the Figure 2, no positional cue was available for the subjects as the stimuli are in front of a blue sky. In pretests, it was discovered that a more realistic condition stabilizes the perception of binocular depth. The torus form around the stimuli was added in order to produce a consistent depth perception for the subjects as this object extends largely in depth. The background was displayed at the maximum possible distance to the observers (depth value equals zero, therefore black).

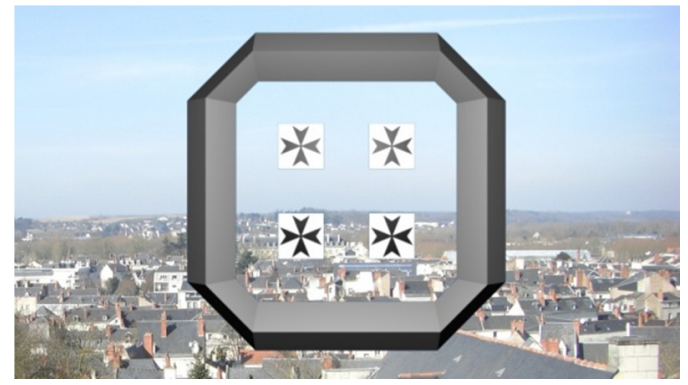

Texture

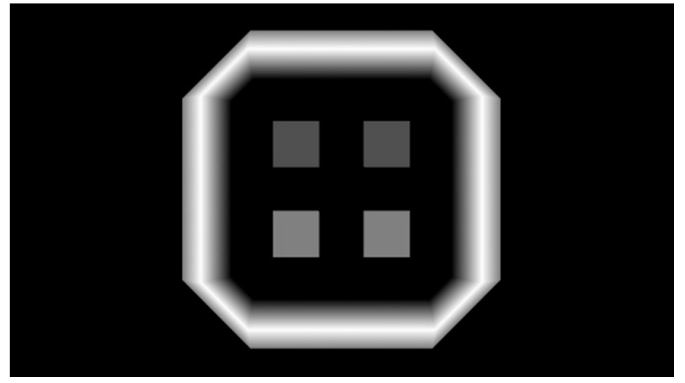

Depth, black equals background

Figure 2: Content displayed on the screen for the depth accuracy experiment

\subsection{Depth experiment}

In the depth experiment, the two upper stimuli showing the Maltese cross were located at different depth. One of the two stimuli had a depth of 80 while the other one varied with depth values larger than 80 . In each trial, a random choice was performed, whether the fixed stimulus was displayed to the left or to the right. The observer was asked to indicate which of the stimuli was closer to the background, e.g. further away from them..

A standard 3up-1down staircase method was used in the experiment with each presentation having a maximum displayed duration of five seconds. The forced choice method was used. Visual feedback about the correctness of the answer was provided to the observers as we were interested in the minimum perceivable depth offset.

The initial stepsize for the depth value decrement was set to two and was successively halved after forty trials. This was done six times, so that in each depth measurement, each observer viewed 240 conditions with the smallest increment being 1/16. The first 6 out of the 240 conditions were discarded for training.

In order to analyze the experimental data from this experiment later on, several preconditions regarding the display need to be known. For this experiment, the settings of the screen were left at the default setting for "signature content". While the rendering system of the Philips screen automatically determines the disparity of the objects based on the values of the depth map, for our analysis it is important to know exactly the disparity of the stimulus on the screen. Several test stimuli were displayed on the screen at different depth and using a macro-capable camera, we were able to determine for each setting the corresponding disparity in pixels and in meters in between two adjacent views. The disparity on the screen $d$ measured in millimeters in function of the transmitted depth value $\mathrm{v}$ was thus empirically determined to correspond to: 
$d=-0.036 \cdot(v-128)$. This is the distance between two corresponding points on the screen, e.g. in x-direction. The resulting functions in terms of visual angle $\alpha$ and absolute distance to the screen $f$ in function of the 8-bit depth value $v$ used in the transmitted depth map are displayed in Figure 3. The distance to the screen specifies the virtual position of the object in front (negative value) or behind (positive value) the screen, thus in z-direction. Please note that the screen has a fixed viewing distance of $g=3.5 \mathrm{~m}$ due to its lenticular array geometry. An average inter-ocular distance of $e=65 \mathrm{~mm}$ was used.

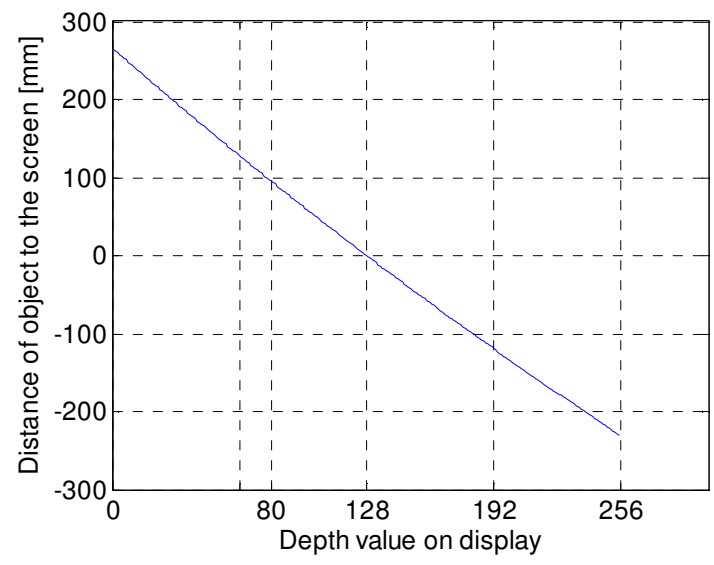

Visual angle: $\alpha=2 * \tan ^{-1}\left(\frac{e-d}{2 g}\right)$

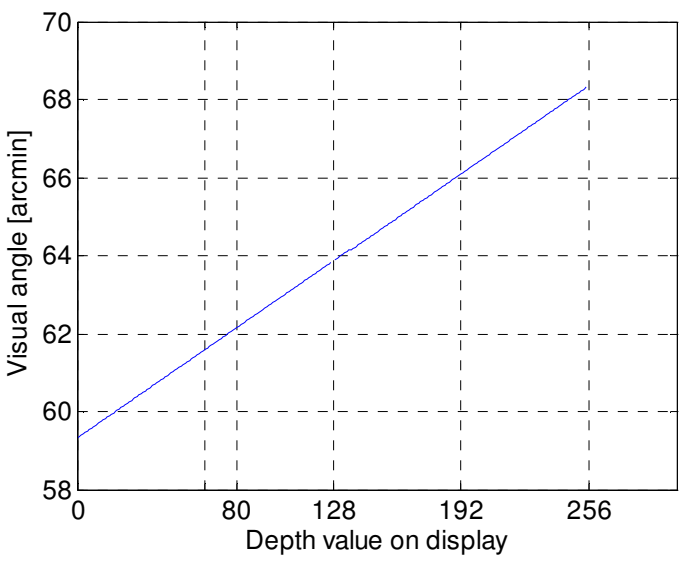

Distance to the screen $[\mathrm{m}]: f=\frac{d g}{e-d}$

Figure 3: Visual angle and the absolute distance to the screen in function of the transmitted depth value

As can be seen, both the visual angle and the distance of the displayed objects to the screen behave mostly linear to the transmitted depth value which simplifies the analysis of the results later on. The linear gradients correspond to approximately 2.1 arcsec/depth-value and $-2 \mathrm{~mm} /$ depth_value.

\section{RESULTS AND DISCUSSION}

\subsection{Fusional vergence facility test at near}

The number of cycles per minute that the observers were able to perform with the two prism settings was recorded for the dominating eye and the non-dominating eye. The results are depicted in Figure 4. On the left hand side, the dominant eye for each observer is examined before and after each of the two sessions. It can be seen that most observers were able to do about 20 cycles per minute but two observers were significantly slower with one not being able to perform the fusion at all. On the right hand side the additional number of cycles after each session is displayed. As can be seen, both after $2 \mathrm{D}$ excitation and after $3 \mathrm{D}$ excitation, the observers performed the optometric test faster than at the start of the session.

For the dominating eye, in 6 cases the increase after 3D viewing was larger than after 2D viewing which is particularly visible for the observers 2, 6, 9 and 10 with respectively 4, 8, 12 and 7 additionnal cycles for 3D compared to $2 \mathrm{D}$. The observers 5 and 8 showed a slightly larger increase after 2D viewing than after 2D viewing with 3 and 2 cycles more. It should be noted that the onetime counting of the number of cycles for one minute in the optometric test involves a certain quantization error as the minute usually finishes within performing a cycle.

For the non-dominating eye, the distinction between $2 \mathrm{D}$ and $3 \mathrm{D}$ viewing is not clearly visible. Four observers showed a higher increase after 2D viewing while another four observers showed a higher increase after 3D viewing. However, in both cases, the number of additional cycles is very small, the maximum being 5 for observer 6 in preference of the 3D session. 


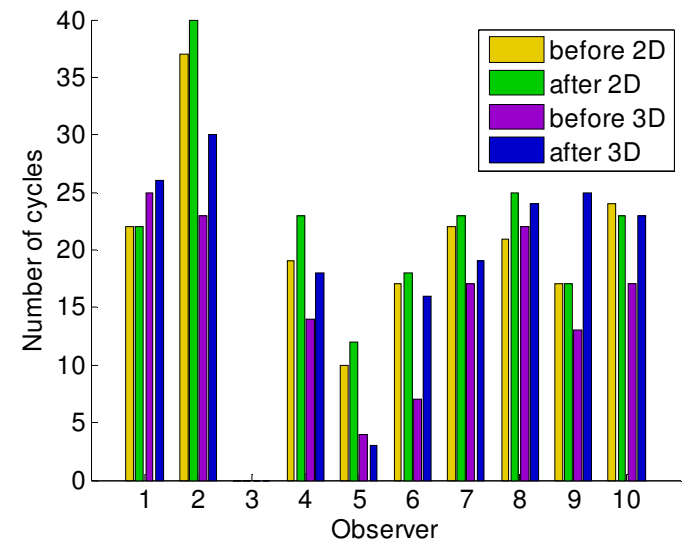

Number of total cycles performed on dominant eye before and after 3D excitation

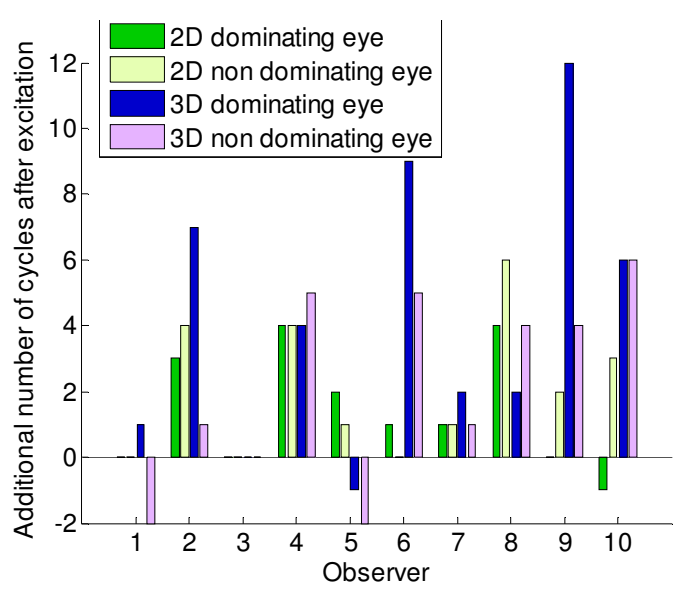

Number of additional cycles performed after 2D or 3D excitation for the dominant and non-dominant eye

Figure 4: Number of cycles performed at the fusional facility test per observer

\subsection{Depth plate test}

The result of the subjective experiment using the depth plates consists of 240 Forced-Choice Paired Comparison decisions per observer. As the first six trials were disregarded for training, the analysis was done with 234 data points. The number of correct decisions is counted for each disparity value displayed on the screen. When using a quantization step size for the disparity data of one, this leads to the bar chart shown in Figure 5. Although the data is noisy, it is possible to fit a cumulative Weibull function as shown in this example using Matlab and the Psychophysics Toolbox extension [15]. A second fit using the Quest algorithm was performed in addition. Both results are shown in the figure. The diagram also shows the $75 \%$ and $92 \%$ points of correct detection for both function fits. In the remaining paper, the discussion will focus on the Weibull function fit.

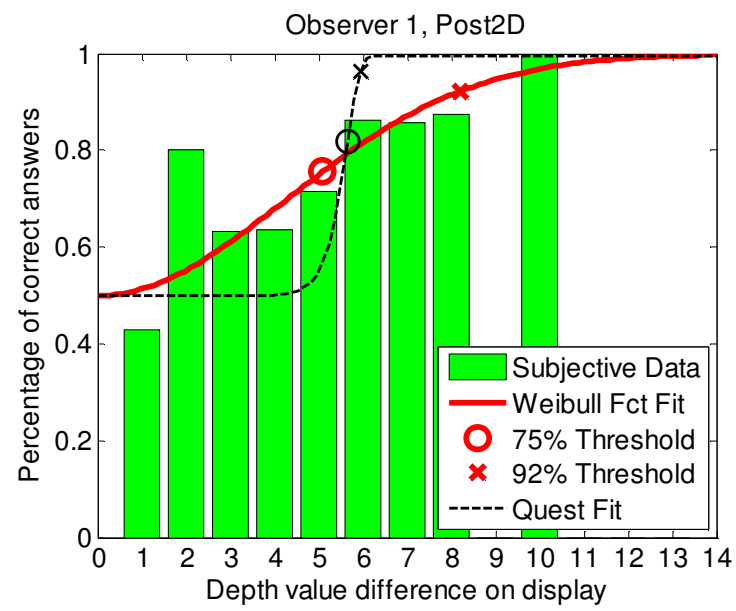

Figure 5: Example for function fit on the experimental data using the Weibull function

From those functions, the $75 \%$ point of correct detection can be determined for each observer before and after $2 \mathrm{D}$ and $3 \mathrm{D}$ viewing, leading to four values per observer. In Figure 6 it can be seen that the $75 \%$ threshold is reached on average with a difference in depth of 3.7 levels while the maximum is at 6 levels. For the $92 \%$ detection threshold, the mean value is at approximately 6.0 depth values with the maximum close to 10 . 


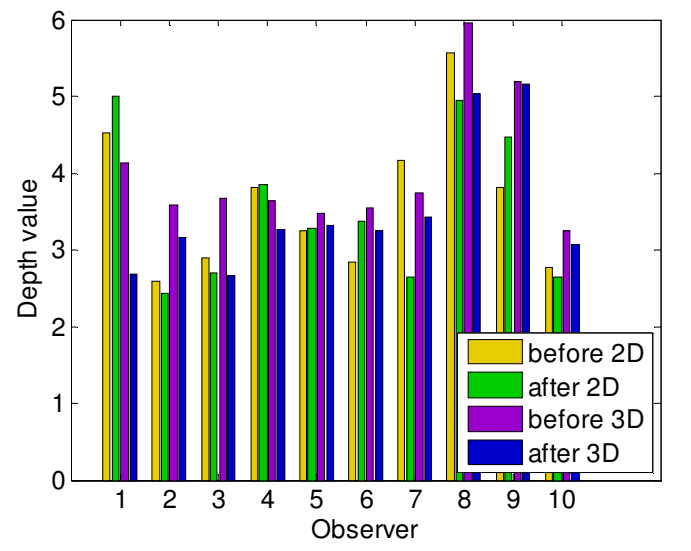

Necessary depth value for $75 \%$ correct detection

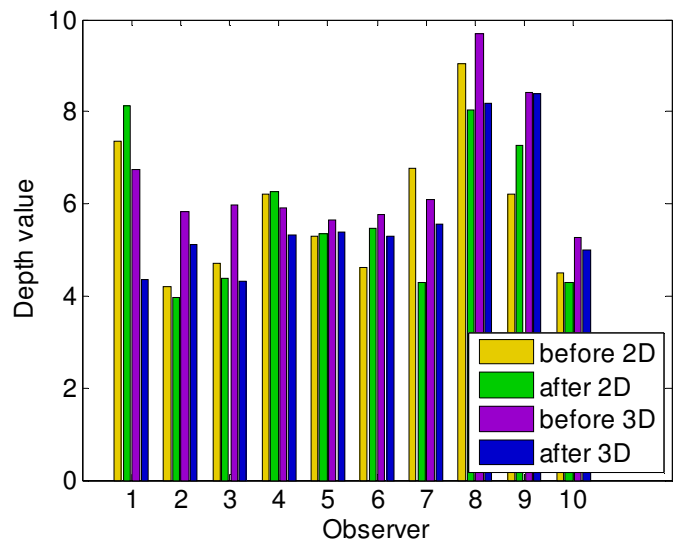

Necessary depth value for $92 \%$ correct detection

Figure 6: Results of the function fit with the cumulative Weibull function at $75 \%$ and $92 \%$ correct detection threshold

Using the results presented in Section 2.3, showing that on this display, the visual angle is approximately linear to the depth value with a gradient of $2.1 \mathrm{arcsec} / \mathrm{depth}$-value, the average value for $75 \%$ detection corresponds to 7.8 arcsec and a correct detection of $92 \%$ is achieved at 12.6 arcsec.

These values are at the lower end of the range of the stereo acuity reported in the literature, e.g. 20 arcsec have been reported in [16]. This result is surprising as it was anticipated that the large amount of crosstalk on the display would render the detection task more difficult. In [13] a similar experiment was performed on the same display but with a different display setting: The depth range was compressed by a factor of about 1.7. The authors reported that about 20 depth levels are required to see a difference which would correspond to about 12 depth levels in our case. As they used an automatic staircase method that is likely to provide a higher detection threshold, the results can be considered coherent.

In order to compare the subject's behavior for the 2D and 3D viewing sessions, Figure 7 displays the difference for the $75 \%$ and the $92 \%$ detection threshold. In contrast to Figure 6, the ordinate directly provides the accuracy gain measured in arcsec. It is obvious that for all except one observer, the accuracy increase was higher after the 3D session than after the $2 \mathrm{D}$ session. For the $92 \%$ threshold, the average value for the $2 \mathrm{D}$ case is -0.3 arcsec indicating a slight decrease of the threshold while for the 3D case, an average of 2.3 arcsec is obtained. Under the assumption that the observeracuity follows a Gaussian distribution, a Student T-Test can be conducted to compare the mean values of the two distributions. It is found that there is a significant difference between the $2 \mathrm{D}$ case and the $3 \mathrm{D}$ case on a $95 \%$ confidence level for the 92\% threshold but not in the $75 \%$ threshold. However, the number of subjects should be considered as small.

These results indicate that there is an increase in accuracy after having watched 3D content on the same screen. One drawback of the experiment conducted so far is that the Philips autostereoscopic screen induces a lot of crosstalk and that the rendering process might introduce artifacts that facilitate or harden the task. It should also be noted that for all observers the first session was the 3D session and thus, they might have performed differently at the very first presentation. However, with 234 depth trials this can be rejected and no indication has been found in Figure 6.

The results also indicate a very high stereo acuity compared to the literature, in particular when the experimental setup is taken into consideration. It should be noted that the measured stereo acuity of 12.6 arcsecs is higher than the typical value for the spatial acuity of the human observer which is in the range of 60 arcsecs for a 20/20 observer. This might indicate that the horizontal resolution of a 3D display needs to be higher than the vertical resolution in order to provide the correct disparity information on the location of objects in depth while the texture information has a lower cut-off frequency. 


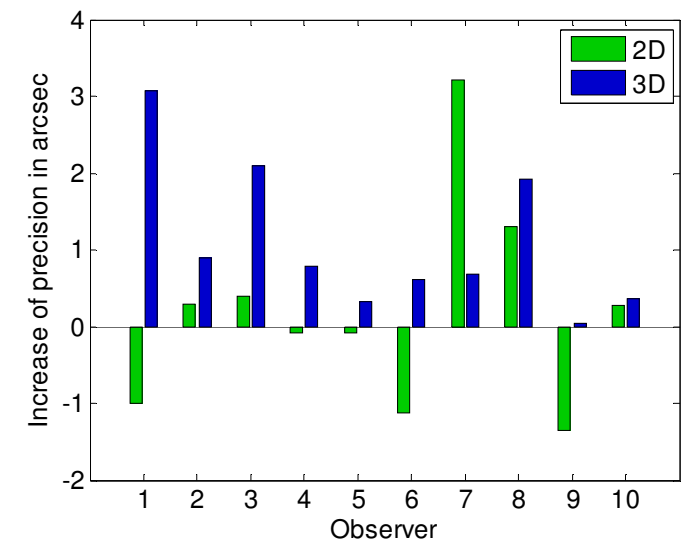

Accuracy increase for $75 \%$ correct detection [arcsec]

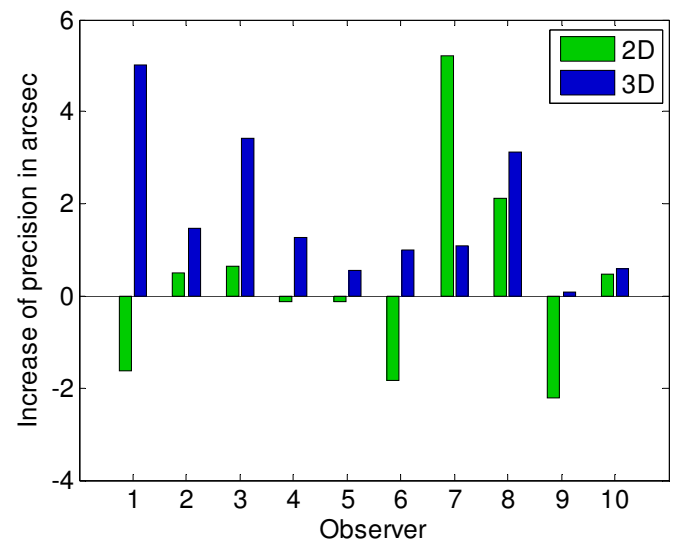

Accuracy increase for $92 \%$ correct detection [arcsec]

Figure 7: Comparison of the depth acuity before and after viewing 2D and 3D respectively

\section{CONCLUSIONS}

In this study, a psychometric experiment has been conducted on a Philips autostereoscopic display in order to find a way to measure differences between a 2D excitation session and a 3D excitation session. It was found that observers were able to detect disparity differences close to and below the acuity threshold previously reported in literature. It was also seen that the $3 \mathrm{D}$ excitation session did not reduce the acuity due to visual fatigue but actually increased the acuity significantly. This effect was not seen in the $2 \mathrm{D}$ case.

It was also possible to verify that the fusional vergence test at near for the dominant eye shows a higher improvement for the $3 \mathrm{D}$ session than for the $2 \mathrm{D}$ session, probably because of the ability of the observers to decouple their accomodation/vergence system during the 3D session.

Thus, two measurements were identified which show objectively that $2 \mathrm{D}$ and $3 \mathrm{D}$ viewing on the same screen affects differently the fusional vergence facility and a measurement of depth acuity performed on the same screen. This difference might be in relation with visual fatigue as observers reported symptoms of visual fatigue after the $3 \mathrm{D}$ session. A larger panel of observers is however necessary to quantify the relationship between the measurements and the visual fatigue.

In particular, there are two not necessarily conflicting hypothesis that need to be investigated. In the following, it is assumed that some adaptation process took place for the observers in the $3 \mathrm{D}$ case and that this adaptation process is inevitable for long-term viewing on this type of screen. The first hypothesis would state that those observers which adapted only partially were experiencing the negative effects of $3 \mathrm{D}$ viewing, e.g. the accommodation-vergence conflict, for a long time. This would lead to higher accumulated visual fatigue with content duration. In this case, the observers with a smaller difference in terms of prism cycles or depth acuity between 2D and 3D viewing would have experienced more visual fatigue. The second hypothesis would state that the adaptation process itself is tiring for the observers. In this case, the observers that showed a larger difference between the $2 \mathrm{D}$ and the 3D case would have felt more visual fatigue. It is often reported that strong visual fatigue is reported only for the first few times when people start viewing $3 \mathrm{D}$, so this would provide a possible explanation.

As the autostereoscopic display used in this experiment has many drawbacks, the next step will be to reproduce the experiment using a stereoscopic screen for the 2D and 3D sessions and a stereoscope for the depth acuity experiment. 


\section{REFERENCES}

[1] Yano, S., Ide, S., Mitsuhashi, T. and Thwaites, H. "A study of visual fatigue and visual comfort for 3D HDTV/HDTV images," Displays 23(4), 191-201 (2002).

[2] Yano, S., Emoto, M., and Mitsuhashi, T. "Two factors in visual fatigue caused by stereoscopic HDTV images," Displays 25(4), 141-150 (2004).

[3] Emoto, M., Niida, T., and Okano, F. "Repeated vergence adaptation causes the decline of visual functions in watching stereoscopic television," Journal of Display Technology 1(2), 328-340 (2005).

[4] Speranza, F., Tam, W. J., Renaud, R., and Hur, N., "Effect of disparity and motion on visual comfort of stereoscopic images," In Proc. of SPIE Stereoscopic Displays and Virtual Reality Systems XIII 6055, (2006).

[5] Li, H.-C.O., Seo, J., Kham, K. and S. Lee, "Measurement of 3D Visual Fatigue using Event Related Potential (ERP): 3D Oddball Paradigm," 3DTV Conference: The True Vision - Capture Transmission and Display of 3D Video, 213-216 (2008).

[6] Lambooij, M.T.M., IJsselsteijn, W.A., and Heynderickx, I. "Visual Discomfort in Stereoscopic Displays: A Review," Stereoscopic Displays and Virtual Reality Systems XIV 6490, (2007)

[7] Kuze, J. and Ukai, K. "Subjective evaluation of visual fatigue caused by motion images," Displays 29(2), 159166 (2008).

[8] Lambooij, M., Fortuin, M., IJsselsteijn, W.A. and Heynderickx, I. "Measuring Visual Discomfort associated with 3D Displays," Proceedings of SPIE - Stereoscopic Displays and Applications XX 7237, (2009)

[9] Barkowsky, M. and Le Callet, P. "The influence of autostereoscopic 3D displays on subsequent task performance," Stereoscopic Displays and Applications XXI 7524-34, (2010).

[10]Ukai, K. and Howarth, P.A. "Visual fatigue caused by viewing stereoscopic motion images: Background, theories, and observations," Displays 29(2), 106-116 (2008).

[11]Fukushima, T., Torii, M., Ukai, K., Wolffsohn, J. S., and B. Gilmartin. "The relationship between CA/C ratio and individual differences in dynamic accommodative responses while viewing stereoscopic images," Journal of Vision 9(13), (2009)

[12] Carlson, N. B., and Kurtz, D. [Clinical procedures for ocular examination], McGraw-Hill (3rd ed.), (2004).

[13]De Silva, D. V. S. X., Fernando, W. A. C. Nur, G., Ekmekcioglu, E., and Worrall, S. T. "3D video assessment with Just Noticeable Difference in Depth evaluation," In 17th IEEE International Conference on Image Processing (ICIP), 4013-4016 (2010).

[14]Barkowsky, M., Cousseau, R., and Le Callet, P. "Influence of Depth Rendering on the Quality of Experience for an Autostereoscopic Display," International Workshop on Quality of Multimedia Experience, (2009)

[15]Brainard, D. H., "The Psychophysics Toolbox," Spatial Vision 10, (1997), see also: http://psychtoolbox.org

[16] Schor, C. M., and Wood, I. "Disparity range for local stereopsis as a function of luminance spatial frequency," Vision Research 23(12), 1649-1654 (1983) 